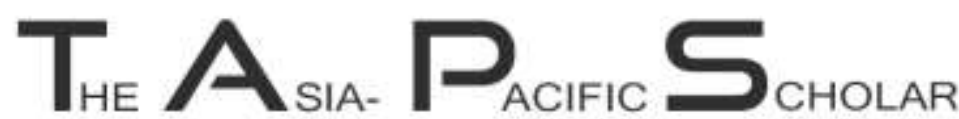

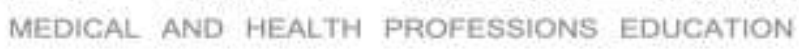

ORIGINAL ARTICLE

\section{A cross-sectional survey of interprofessional education across 13 healthcare professions in Japan}

\author{
Michiko Goto ${ }^{1}$, Junji Haruta ${ }^{2}$, Ai Oishi ${ }^{3}$, Kazue Yoshida ${ }^{4}$, Kenji Yoshimi ${ }^{5}$, \\ Yousuke Takemura ${ }^{1,6}$ \& Hisashi Yoshimoto ${ }^{7}$
}

${ }^{1}$ Department of Education and Research in Family and Community Medicine, Mie Univers ity Graduate School of Medicine, Tsu, Japan; ${ }^{2}$ Department of General Medicine and Primary Care, University of Tsukuba Hospital, Tsukuba, Japan; ${ }^{3}$ Primary Palliative Care Research Group, Usher Institute of Population Health Sciences and Informatics, Univers ity of Edinburgh Medical School, Edinburgh, UK; ${ }^{4}$ Department of Adult Health and Psychiatric Nursing, Faculty of Mie University, Tsu, Japan; ${ }^{5}$ Department of Contemporary Sociology, School of Sociology, Bukkyo University, Kyoto, Japan; ${ }^{6}$ Department of Family Medicine, Mie University School of Medicine, Mie, Japan, ${ }^{7}$ General Medicine and Primary Care, University of Tsukuba, Faculty of Medicine, Tsukuba, Japan

\begin{abstract}
Background: With the rapid aging of the population, collaboration among the various medical professions throughout the country is becoming increasingly indispensable in Japan. To promote collaboration in medical care, it is necessary to introduce the c oncept of collaboration to students at an early stage of their professional education. Despite this need, there are no core medical, healthcare and welfare education curricula in Japan that include interprofessional education(IPE). Therefore, the status of IPEin Japanese schools of medicine and other healthcare-related services is unclear.

Methods: We sent 3,430 questionnaires to 13 schools of medicine and related healthcare providers: doctors, nurses, physical therapists, registered dieticians, dentists, dental hygienists, social workers, pharmacists, occupational therapists, clinical psychologis ts, medical adminis trators, certified care workers, and speech therapists. The items addressed implementation rate of IPE, teaching methods and grading methods.

Results: Of the 3,430 surveys dis tributed, 572 completed surveys were returned (response rate $17 \%$ ). Of the completed surveys, 493 qualified as valid, resulting in a final response rate of $14 \%$. Only $19 \%(\mathrm{n}=93)$ of the analysed medical educational ins titutions included IPE instruction in their curricula.

Conclusion: While many educational institutions were conducting IPE, there were important inter-institutional differences with respect to attitudes towards IPE, teaching methods, and evaluation methods. This study was unprecedented in scale, and provides important basic information for the future development of IPE in Japan.
\end{abstract}

Keywords: $\quad$ InterprofessionalEducation, Healthcare Professions, Teaching Methods, Grading Methods, Collaboration

Practice Highlights

- IPE is being implemented in about $19 \%$ of the educational institutions contacted, and is represented in 13 medical, healthcare and social professions.

- Of the 13 represented professions, all implemented IPE in at least some of their participating schools.

- The greatest proportion (68\%) of IPE was delivered through group learning.

- Essays were the most frequent (65\%) evaluation method used in IPE.

\section{INTRODUCTION}

The Japanese population is aging at an unparalleled rate, whereas birth rates are declining (Cabinet Office, 2011). The population aged 65 years and older is now over 30 The Asia Pacif ic Scholar, Vol. 3, No. 2 / May 2018 Copy right @ 2018 TAPS. All rights reserved. million. The Ministry of Health, Labour and Welfare estimates that the population aged 65years and older in Japan will peak at approximately 39 million people in 2042, and the percentage of the population that is 75 
years or older will continue to increase after that point. Therefore, by 2025, the ministry aims to establish an Integrated Community Care System (ICCS) comprising medical care, nursing care, disease prevention, housing, and support in daily living (Ministry of Health, Labour and Welfare, 2016). This system would enable older people to live out their lives in their local communities according to their own values.

Because the ICCS is interprofessional by nature, there is a need for professionals who can promote collaboration among medical, healthcare, and welfare services (Hughes et al., 1992; Janssonc, Isacsson \& Lindholm, 1992; Lemieux-Charles \& McGuire, 2006). Training of professionals with such abilities is urgently required. Under the motto "learning together," the World Health Organization stresses the importance of training health workers who can respond to the health needs of the people they serve while working in an interprofessional team to optimise available resources (Gilbert, Yan, \& Hoffman, 2010; World Health Organization [WHO], 1988). To achieve this, education within individual specialties is insufficient. Rather, it is essential that the idea of collaborating with various specialties is introduced into the early stage of students' professional education (Subcommittee on Elderly Health, Committee on Health/Human Life Science, Science Council of Japan, 2011). The need for such human resource development is not a unique is sue to Japan; it is a global issue considering that the population aging is a worldwide problem.

The UK and Australia have well-known examples of advanced interprofessional education (IPE) (Barr, Marion, D'Avray, \& Thistlethwaite, 2013; The Interprofessional Curriculum Renewal Consortium, Australia, 2013). In Asia, Singapore's population is aging almost as rapidly (estimated at $22.3 \%$ by 2025 ) as Japan's (Oizumi, 2007), and authorities at the National University of Singapore are reportedly developing IPE (Jacobs et al., 2013). In Japan, similarly, Saitama Prefectural University launched IPE modules in 2006, and Gunma University and 10 health and welfare higher education institutions initiated IPE in 2008 (Japan Interprofessional Working and Education Network [JIPWEN], 2016). Because of the activities of these leading institutions, the number of health and welfare schools that have implemented IPE is growing. However, IPE is not yet regarded as compulsory in Japanese health-related profes sional education.

Langton (2009) has identified five models of IPE among British institutions of higher education. However, it is not clear how IPE is carried out in these models and little is known about what kind of curriculum is being implemented. Two relatively large-scale surveys have already been published in Japan (Oshima, 2009; Ogawa, Takahashi \& Miyazaki, 2015). One addressed recognition of and potential for IPE development, while the other collected information on the current status of and problems with IPE. The former dealt with five professions (doctor, nurse, physical therapist, occupational therapist, social worker), the latter with eight (doctor, nurse, physical therapist, occupational therapist, social worker, pharmacist, dentist, dietitian). Both of the survey reports were limited to universities; there are no nationwide surveys that include technical colleges (5-year graduate educational institutions including high school level), junior colleges, and vocational schools, all of which are still an essential part of professional education. Vocational schools in Japan provide practical vocational and technical education in 1to 3 -year courses. More than $40 \%$ of graduates from vocational schools work in the health and welfare field with national licenses (Ministry of Education, Culture, Sports, Science and Technology, 2013). However, it is not clear how much IPE is carried out, nor what the teaching methods and evaluation criteria are at such schools.

The present national survey conducted in Japan aims to clarify the current status of IPE, implementation percentages of IPE by profession and school type, and teaching methods and evaluation criteria employed.

\section{METHODS}

\section{A. Questionnaire Development}

We reviewed large-scale surveys conducted in the UK, Canada, Australia, and the US. Our analysis showed that across these countries, there was no unified survey at the national level, and that the contents of the surveys differed depending on the context of the country and institution (Barr et al., 2013; Formicola et al., 2012; International Network Health Policy and Reform, 2011; Rafter et al., 2006; The Interprofessional Curriculum Renewal Consortium, Australia, 2013).

Of the surveys we reviewed, a UK survey of 127 institutions representing 16 professions seemed to be the most appropriate reference for us, given our aim of evaluating the actual situation and curriculum framework of the institutions for healthcare professions. We developed our questionnaire on IPE health and welfare schools in 2013 as follows.

First, a native Japanese speaker translated the questionnaire from the UK survey into Japanese. All the authors reviewed this translation to ensure that it matched the current situation in Japan; a draft version of the questionnaire was then formulated. Next, three college faculty members who were engaged in IPE were asked to complete the draft questionnaire and to provide feedback. The comments and suggestions of the faculty 
members were used to develop the final version of the questionnaire.

The survey items covered the following topics: target specialty or profession; type of school (technical college, vocational school, junior college, university, graduate school of a university); presence or absence of IPE and IPE training; curriculum profile (course title, target profession, instructors, hours, units, teaching method, grading system); course details (outcomes assessment, course review process, research involvement/practice-oriented, joint program with extramural institutions, collaborators, challenges in implementing IPE); and, open-ended comments. Medicine, dentistry, and pharmacy are 6-year programs, were categorized as undergraduate level.

\section{Development of the questionnaire}

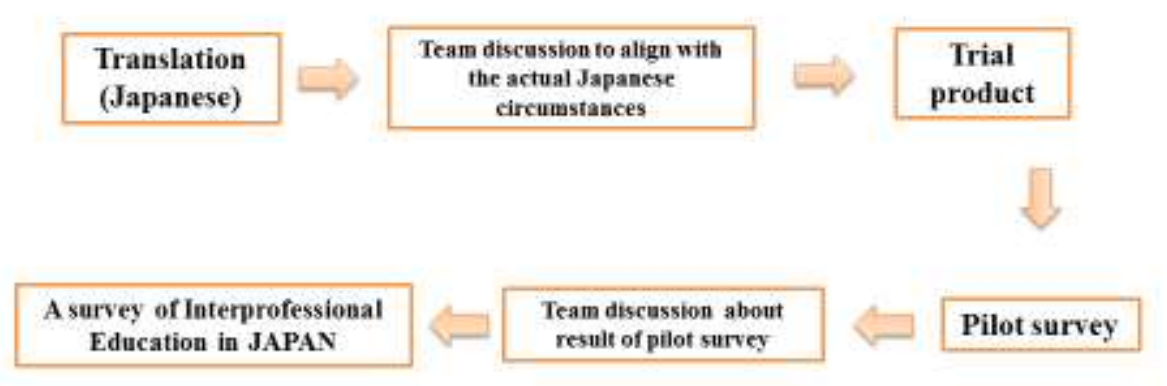

Figure 1. The process for developing the questionnaire on IPE

\section{B. Participants and Recruitment}

The questionnaire was sent to the chief administrative officers at 3,430 health and welfare schools, covering 13 healthcare and social care professions, where medical professionals who are a key part of the ICCS are trained in Japan: doctors, nurses, physical therapists, registered dietitians, dentists, dental hygienists, social workers, pharmacists, occupational therapists, clinical psychologists, medical administrators, certified care workers, and speech therapists. Except for medical schools, the schools chosen to receive questionnaires were those listed on the websites of the academic societies of the aforementioned professions (Yodosha Meibo Henshushitsu, 2013). To choose medical schools, we used a directory of medical schools.

The questionnaire was mailed on December 18, 2013, along with an informed consent form, cover letter, and a return envelope, to the chief administrative officers in charge of curriculum management at each school. The closing date of the questionnaire was set as January 28, 2014.

\section{Data Analysis}

The data were screened by two researchers (MG and AO) and analysed using Excel 2013 software (Microsoft Japan, Tokyo, Japan). We did not analyse statistically.
Summary data are presented as frequencies and percentages.

The research protocol of this study was approved by the Research Ethics Committee of the Mie University School of Medicine.

\section{RESULTS}

In total, 572 completed questionnaires were returned (response rate $17 \%$ ), of which the number of valid responses was $493(14 \%)$. 3\% were excluded because the department was not identifiable.

\section{A. Implementation Rate of IPE Curriculum}

The percentage of the responding schools that provided IPE was 19\% (93 of 493), with a total of 236 curricula, a median of 22.5 hours, and a quadrant range of 10 to 40 hours.

The following professions are listed in order of IPE implementation rate: speech therapy $60 \%$; dentistry $55.6 \%$; medicine $37.5 \%$; occupational therapy $31.3 \%$; pharmacy 29\%; clinical psychology 29\%; dental hygiene $23.4 \%$; social work $20 \%$; physical therapy $16 \%$; nursing 13.6\%; medical secretarial $13.3 \%$; certified care work $12.1 \%$; and nutrition $11.1 \%$. There were no occupations in which IPE was completely absent (See Table 1). 


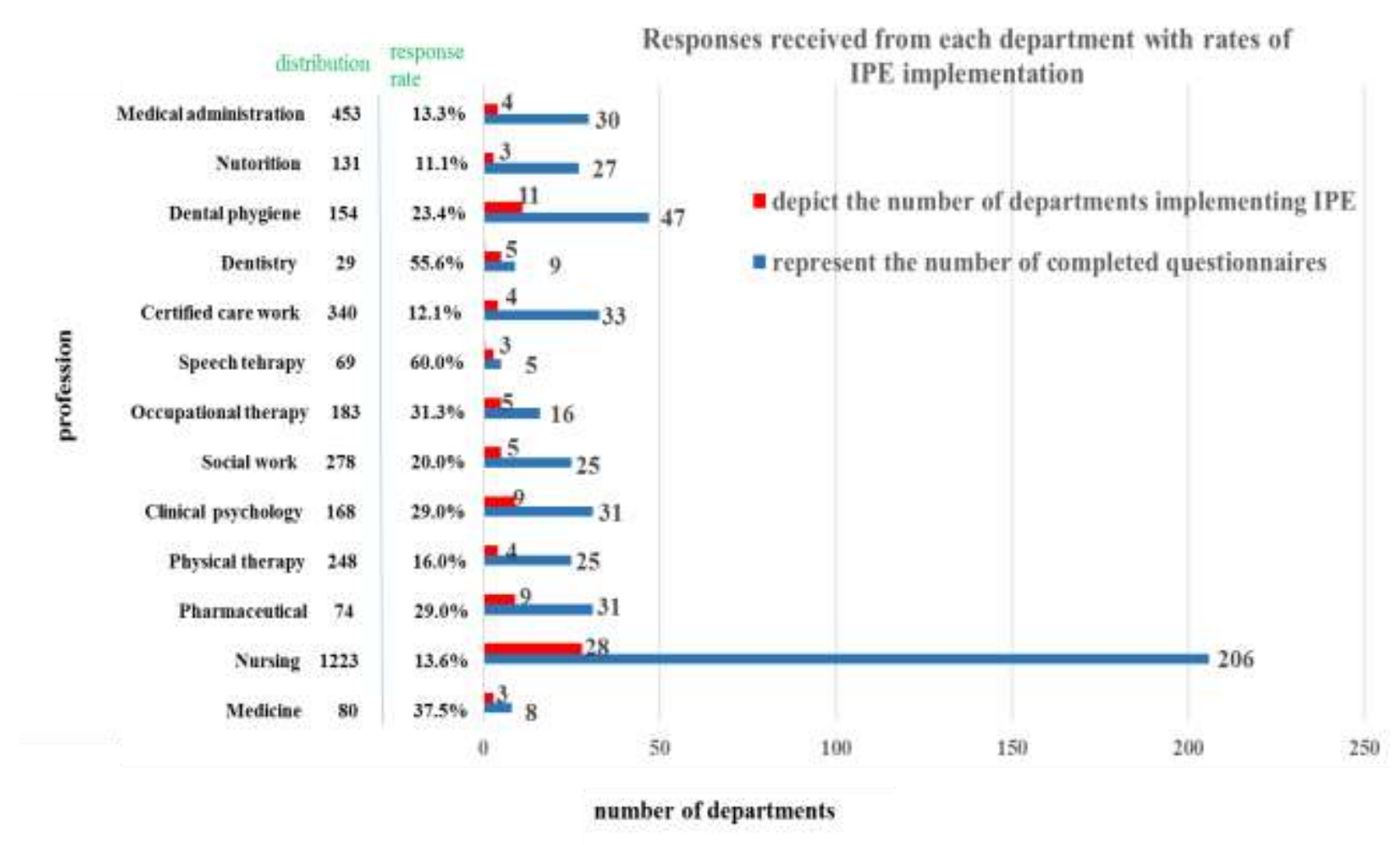

Note: Distribution represents the number of questionnaires distributed to departments Table 1. Responses received from each department with percentages of IPE implementation

\section{B. Implementation by School Type}

The number of valid responses and the institutions providing IPE courses or modules are shown in Table 2. Universities providing undergraduate degrees had the highest percentage $(33 \%)$ of IPE within their curricula, followed by those with graduate degrees (28\%), 3-year vocational schools (14\%), junior colleges (9\%), technical colleges (7\%), and 2-year vocational schools $(5 \%)$.

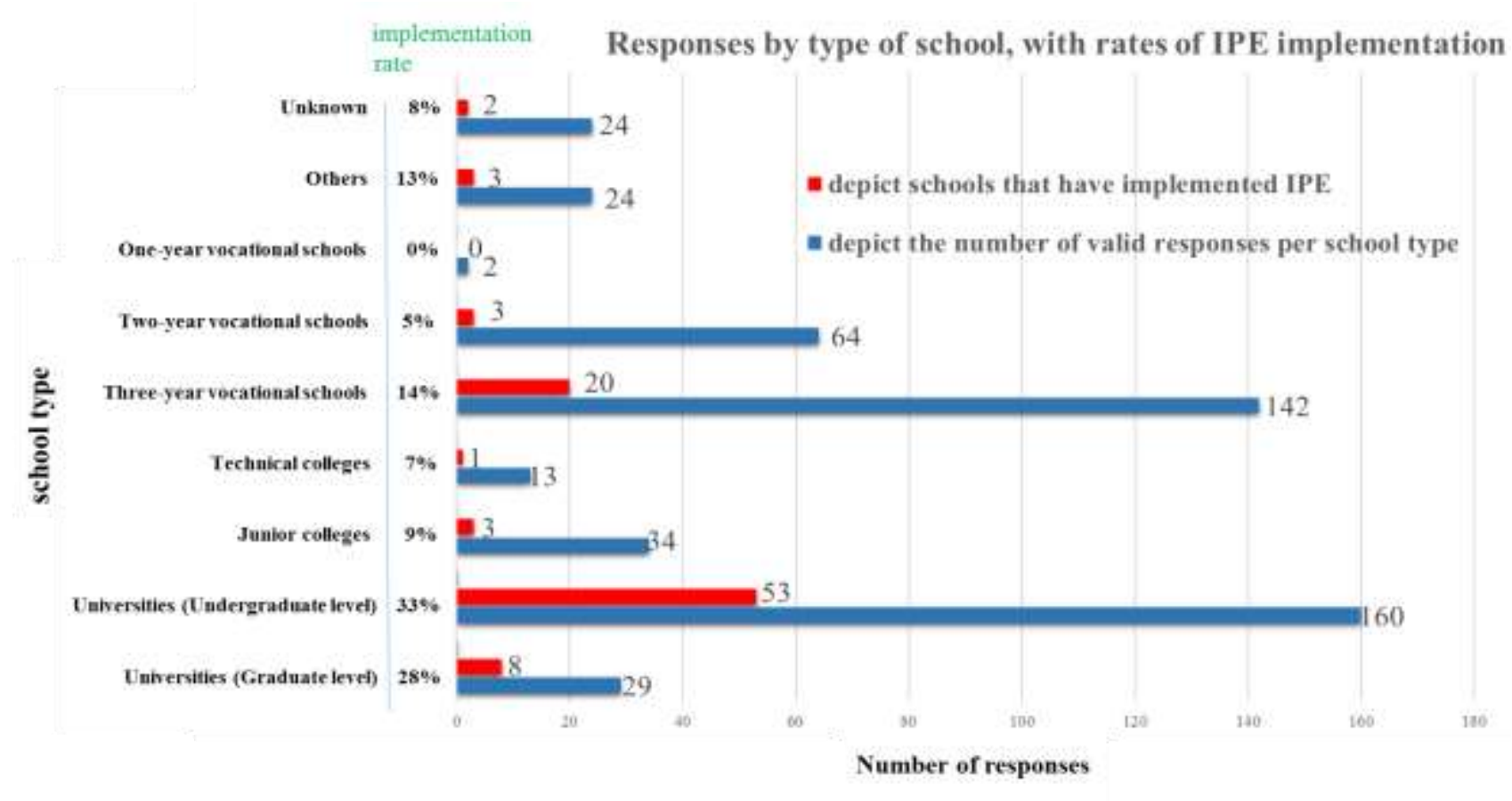

Table 2. Responses by type of school, with percentages of IPE implementation

\section{Teaching Methods}

Teaching methods in 244 courses that included IPE at 93 institutions were analysed. The results are shown in Table 3. The greatest proportion of IPE was delivered The Asia Pacific Scholar, Vol. 3, No. 2/ May 2018 Copy right @ 2018 TAPS. All rights reserved. through group learning (e.g., case-based learning, problem learning, practice learning, experiential learning, or role-play exercises; 68\%), followed by lectures (48\%). Lectures alone represented $21 \%$ of the 
answers; only three courses or modules included elearning $(1 \%)$.

\section{Teaching methods}

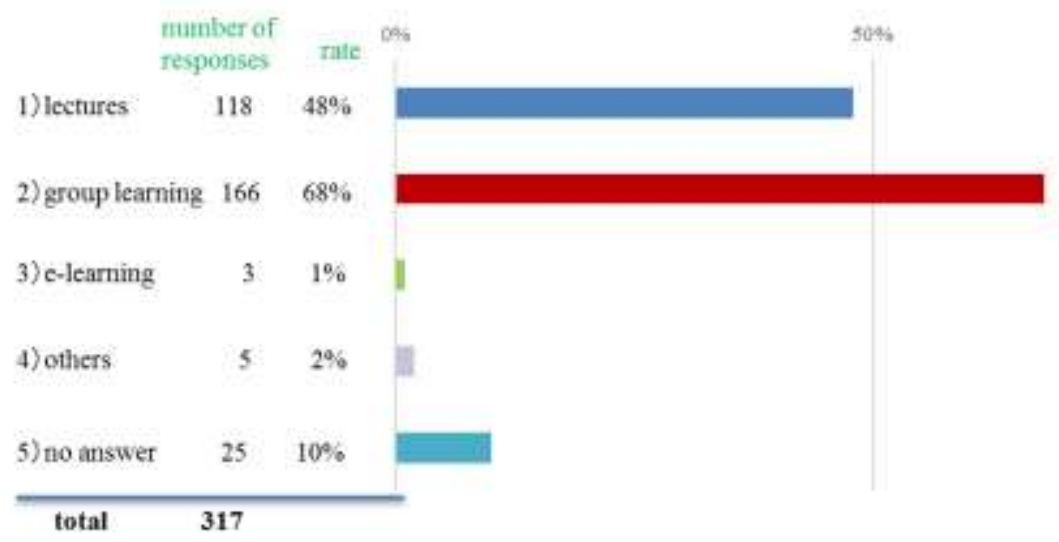

Table 3. Teaching methods used for the delivery of IPE

\section{Grading Methods}

Of 244 grading systems reported (multiple answers allowed), essays were the most frequent evaluation method (65\%), followed by paper exams (25\%), portfolios (9\%), and Objective Structure Clinical Examinations (OSCE) (6\%). Other methods included attendance, peer review, poster presentations, oral presentations, self-evaluation, and interviews.

\section{E. Interest in IPE Survey Results}

The majority of respondents $(n=306,62 \%)$ indicated interest in knowing the outcome of this survey.

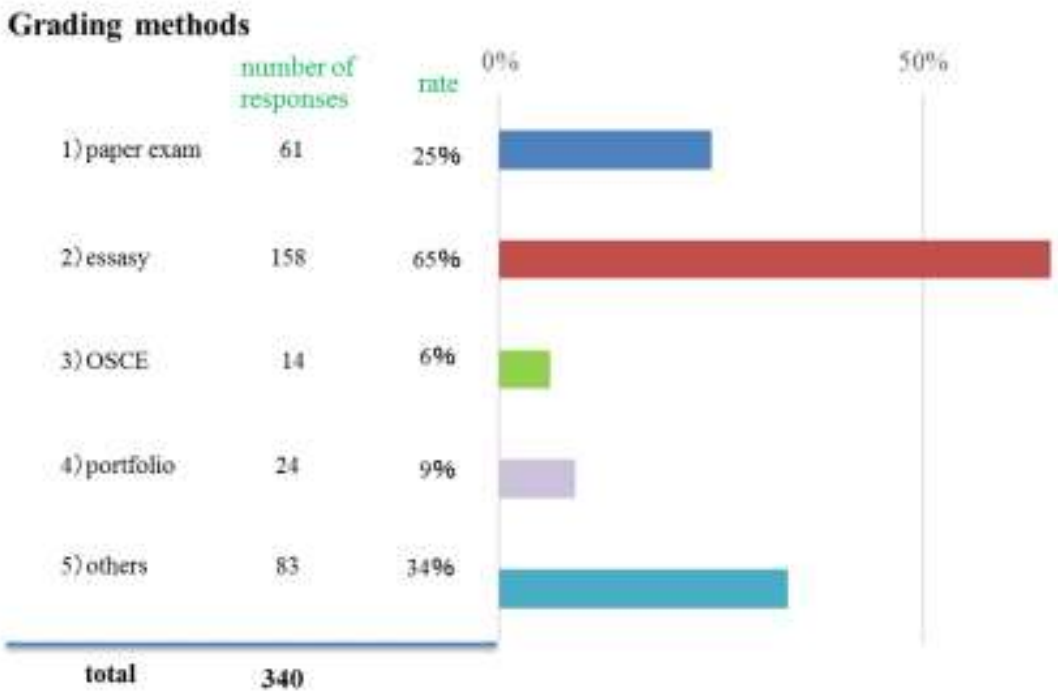

Table 4. Grading methods used for IPE

\section{DISCUSSION}

Our results showed that $19 \%$ of the health and welfare schools surveyed provided IPE courses or modules. However, the rate of IPE implementation is not keeping pace with the need for service, because the percentage of the population, 65 years or older, is increasing unprecedently in Japan. (the aging rate in 2010 was 23\%) (Cabinet Office, 2014). Unfortunately, because no implementation frequency surveys have been conducted in the UK or Australia, it is not possible to compare our results with those of other countries (Barr, et al., 2013;
The Interprofessional Curriculum Renewal Consortium, Australia, 2013). To disseminate IPE, which requires considerable resources for coordination with other departments or extramural institutions, it may be necessary to boost organizational support at the institutional level or at the policy-making level for all health and welfare institutions.

Our survey showed that IPE was most prevalent in universities (undergraduate level), which had higher implementation percentages than did specialized training 
schools. This is arguably related to the freedom that universities have in organizing the curriculum of their 4year undergraduate education, through which they can reflect their ideas regarding both their specialized and liberal arts fields (Hayashi, 2003; Ministry of Education, Culture, Sports, Science and Technology, 2001). Conversely, the implementation of IPE in vocational schools may be affected by these schools' focus on obtaining professional qualifications within a shorter timeframe, which may make it more difficult to cover a broader range of subjects (Tsukahara, 2005).

This survey revealed the difficulty of practicing IPE at vocational schools. However, some specialized vocationalschools are now improving or expanding their curricula to 4 years and making it easier to obtain qualifications (e.g., nurses can get a health nurse or midwifery license), which improves the outlook for the introduction of IPE in the future. In 4-year curricula, the proportion of total class hours dedicated to practical training and exercises exceeds $40 \%$ in the medical field; there is great potential for introducing IPE in the future if the practical training in multi-professional curricula can be capably coordinated (Ministry of Education, Culture, Sports, Science and Technology, 2013). We speculate that the key to introducing IPE in vocational schools will be the way in which the practical training or exercise is used.

Our results for teaching methods showed that $68 \%$ of the curricula incorporated some form of group learning approach, $48 \%$ included lectures, and $21 \%$ were based on lectures alone. Abu-Rish reported that in the 83 programs studied, $57.8 \%$ were small group discussions, $48.2 \%$ were case-based or question-based learning; and $36.1 \%$ were large-group lecture (Abu-Rish et al., 2012). In the UK study, all the learning methods reported were interactive (Barr et al., 2013). The UK-based Centre for the Advancement of IPE maintains that IPE occurs when two or more professions "learn with, from, and about each other" (Centre for The Advancement of Interprofessional Education, 2017). In light of this definition, it is a heartening result that the interactive strategy is dominant: $68 \%$ of respondents reported use of group learning.

We do not know whether $21 \%$ lectures alone should be interpreted as high figure. While it is necessary to confirm the details of the lecture format, lectures, which simply transfer knowledge from the instructor to the students, may not be sufficiently conducive to collaboration. Lectures are useful as a form of teaching that conveys information to a large number of people at once. Japanese educators may tend to rely on lecturing because they do not have enough knowledge about and experience with active learning, a method in which learners are taking part more deliberately, rather than merely listening to and memorizing the lecture passively. Japan may also have fewer educational environments designed for active learning. However, the Australian survey reported that 21 of the 70 curricula also included lectures; therefore, we do not think that lectures are unnecess ary (The interprofes sional Curriculum Renewal Consortium, Australia, 2013); however, we suggest that lectures will be more useful if they are designed to complement the interactive program.

The Japanese Media Developmental Education Centre has reported that $46.1 \%$ of higher education institutions use IT-based methods such as e-learning (Media Education Development Centre, 2006): Universities use it for $56.7 \%$ of courses, colleges for $24.4 \%$, and special training colleges for $72.1 \%$ (Mima \& Yamaguchi, 2005). In our survey, by contrast, only three courses (1\%) included e-learning for IPE, which is much lower than the 58\% reported in the UK (Barr et al., 2013). Importantly, online learning could enable geographically distant schools to hold joint sessions. It could also as sist in launching an online IPE course or module at institutions that cannot start their own because of a lack of suitable disciplinary combinations (Luke et al., 2009).

The most common method for evaluating IPE learning was the term essay (65\%), followed by the paper exam (25\%). Thistlethwaite and Moran (2010) has suggested that IPE outcome evaluations should focus more on changes in the learners' attitudes or actions than on their knowledge, in which case, self-evaluation is often carried out. Therefore, the essay may be inadequate. For self-reflection and self-evaluation, a portfolio method would be better. Thistlethwaite, Kumar, Moran, Saunders and Carr (2015) reported that in a recent review of pre-qualification IPE evaluations, of the 90 studies included, only 5 reported on assessment of student knowledge via a written test. Abu-Rish et al (2012) reported that $39.8 \%$ of the assessment methods were knowledge-based. But in the Australian survey (The interprofessional Curriculum Renewal Consortium, Australia, 2013), teamwork and team function were also evaluated. Our questionnaire did not ask for details about assessment processes. Future studies should, therefore, investigate how IPE competencies are evaluated with paper exams, as well as determine the extent to which rubrics and other evaluation tools have been introduced.

The programs may use attendance as an assessment tool because appropriate validated as ses sment tools have not been developed yet. Attendance was used for some portion of evaluation in the Australian survey (The Interprofessional Curriculum Renewal Consortium, Australia, 2013). Summative assessment of IPE as attendance may be unavoidable, because prequalification 
professional education focuses on the individual, rather than the collaboration unit. Given the current situation in Japan, where IPE practice has preceded development of appropriate as sessment methods, essays and paper exams have been the major methods of evaluating IPE. As a next step, we must develop valid and reliable tools for such evaluations; this is a challenge for those engaged in IPE not only in Japan, but around the world.

In April 2016, we developed the "Interprofessional competency framework in Japan" (Haruta et al., 2016) to define the skills necessary for collaboration among multiple professional academic associations. Thus, our future tasks include the development of reports and OSCE evaluation methods based on competency for collaboration.

Additionally, we are also conducting a survey with faculties to investigate their awareness, learning environments, outcomes, and evaluations of IPE. We also intend to conduct qualitative and individual research with universities or vocational schools that offer advanced approaches, by visiting the institutions directly and interviewing people in charge of IPE. In this way, we can clarify and disseminate effective learning methods and tips for overcoming the barriers to IPE introduction. Finally, we will compare IPE tips in Western countries with those in Japan.

Given that Japan will implement IPE from now on, our analyses may also be useful for Asian countries with similar cultural backgrounds. Also, developing countries that do not have detailed data or any organizations promoting IPE such as in the UK, Australia and Canada, may find our data collection methods useful. We believe this will result in the spread of IPE not only in Japan but also in many other Asian countries and developing countries.

The implementation rate of IPE in Japan was as low as 19\%; however, our low survey response rate may have affected the results. This low rate may also reflect the inclusion of vocational schools, junior colleges and special training schools. We suspect that the low response rate may have had to do with a failure to reach the appropriate persons in the institutions. Although we sent the survey to the chief administrative officer in charge of curriculum management at each institution, with the expectation that it would be passed to the curriculum director of IPE where such a position existed, this may not have occurred in every instance. Because some courses involved different divisions or departments, and occasional extracurricular sessions, the recipients may have experienced difficulty finding the appropriate instructors. We initially anonymized the survey to facilitate the completion process. We expected that the responses would come from each individual professional but some responses came from administrative offices; in such cases, we were unable to identify the department, and those responses were therefore excluded. In retrospect, we believe it would have been preferable to identify the person in charge of IPE at each institution before sending the questionnaires.

It should be pointed out that the response rates in previous Japanese studies have been similarly low at 24.6\% (Oshima, 2009) and 32.8\% (Ogawa et al., 2010). The UK survey (Barr et al., 2013) mentioned earlier made reference to difficulties in identifying interprofessional curricula, suggesting that a questionnaire-based investigation of IPE has inherent limitations. Our future work will improve this survey, and evolve toward locating responsible institutional contacts to provide regular updates regarding IPE curricula.

\section{CONCLUSION}

IPE is being implemented in about $19 \%$ of the educational institutions contacted, and is represented in 13 occupations. We clarified the current level of IPE in graduate, undergraduate, technical college, junior college, and vocational schools for these 13 major healthcare and social care professions in Japan. This study can provide important fundamental information for the development of IPE curriculum and serve as a springboard from which to develop deeper empirical insights.

\section{Notes on Contributors}

Michiko Goto is an Assistant Professor in Department of Education and Research in Family and Community Medicine, Mie University Graduate School of Medicine.

Junji Haruta is an Assistant Professor in Department of General Medicine and Primary Care, University of Tsukuba Hospital, Japan.

Ai Oishi is a PhD candidate at the Usher Institute of Population Health Sciences and Informatics, University of Edinburgh, UK.

Kazue Yoshida is an Associate professor in Graduate School of Medicine, Course of Nursing, Faculty of Medicine Mie University, Mie, Japan.

Kenji Yoshimi is a Lecturer in Department of Contemporary Sociology, School of Sociology, Bukkyou University, Kyoto, Japan.

Yousuke Takemura is Professor and Chair in Department of Family Medicine, Mie University School of Medicine \& Graduate School of Medicine, Mie, Japan. 
Hisashi Yoshimoto is an Assistant Professor in Department of Primary Care and Medical Education, Faculty of Medicine, University of Tsukuba, Tsukuba, Japan.

\section{Ethical Approval}

This study was approved by the Research Ethics Committee of the Faculty of Medicine at Mie University (No. 1396).

\section{Acknowledgements}

The authors would like to thank all of the participants who cooperated in this questionnaire, and to convey special thanks to Takashima, our office secretary.

\section{Declaration of Interest}

The authors declare that they have no competing interests.

\section{References}

Abu-Rish, E., Kim, S., Choe, L., Varpio L., Malik E., White, A. A., \& Zierler, B. (2012). Current trends in interprofessional education of health Sciences students: A literature review. Journal of Interprofessional Care, 26, 444-451.

Barr, H., Marion, D’Avray, M.H.L., \& Thistlethwaite, J. (2013). A Survey of pre-registration interprofessional education in the United Kingdom 2010-2012. Retrieved from

http://caipe.org.uk/silo/files/ipergsurvey30april2013finalforeword q.pdf.

Cabinet Office (2011). The situation of aging. Heisei era 2011 aged society white paper. Tokyo: Government of Japan. Retrieved from http://www8.cao.go.jp/kourei/whitepaper/w-011/zenbun/html/ s1-1-1-02.html.

Cabinet Office (2014). International trend of aging. Annual Report on the Aging Society: 2014 (Summery). Tokyo: Government of Japan. Retrieved from http://www8.cao.go.jp/kourei/english/ann u alreport/2014/pdf/c1-1.pdf.

Centre for The Advancement of interprofessional Education (2017). Interprofessional Education Guidelines. Retrieved from https://www.caipe.org/resources/publications/caipepublications/caipe-2017-interprofessional-education-guidelinesbarr-h-ford-j-gray-r-helme-m-hutchings-m-low-h-machin-reeves-s.

Formicola, A. J., Andries, S. C., Buchnan, J. A., Childs, G. S., Gibbs, M., Inglehart, M. R., Evans, L. (2012). Interprofessional education in US and Canadian dental schools: An ADEA Team Study Group report. Journal of Dental Education, 76(9), 12501268

Gilbert, J. H. V., Yan, J., \& Hoffman, S. J. (2010). A WHO Report: Framework for action on interprofessional education and collaborative practice. Journal of Allied Health Association of Schools of Allied Health Professions, 39, 196-197.

Haruta, J., Sakai I., Otsuka M., Yoshimoto, H., Yoshida K., Goto, M., \& Shimoi, T. (2016). Development of an interprofessional competency framework in Japan. Journal of Interprofessional Care, 30 (5), 675-677.

Retrieved from https://www.ncbi.nlm.nih.gov/pubmed/27351518.

Hayashi, M. (2003). Problem of general education brought about by the relaxation of standards for the establishment of universities.
Memoirs of the Osaka Institute of Technology (Series B), 48(2), 1326. Retrieved from https://www.oit.ac.jp/japanese/toshokan/tosho /kiyou/jinshahen/48-2/jinsya_2/hayashi_masa hito.html.

Hughes, S. L., Cummings, J., Weaver, F., Manheim, L., Braun, B., \& Conrad, K. (1992). A randomized trial of the cost effectiveness of VA hospital-based home care for the terminally ill. BMC Health Services - Research, 26(6), 801-817.

International Network Health Policy and Reform (2011). Interprofessional Education: A progress report. Retrieved from http://www.hpm.org/en/Surveys/CPRN_-_Canada/12/Interprofess ional_Education___A_progress_report.html.

Jacobus, J. L., Samarasekera, D. D., Chui, W. K., Chan, S. Y., Wong, L., Liaw, S. Y., Chan, Y. C. S. (2013). Building a successf ul platform for interprofessional education for health professions in an Asian university. Medical Teacher, 35(5), 343-347.

Jansson, A., Isacsson, A., \& Lindholm, L. H. (1992). Organization of health care teams and the population's contacts with primary care. Scandinavian Journal of Primary Health Care, 10(4), 257265.

Japan Interprofessional Working and Education Network [JIPWEN] (2016). News Website.

Retrieved from http://jipwen.dept.showa.gunma-u.ac.jp/jp/?cat=6.

Langton, H. (2009). Interprofessional education in higher education institutions: models, pedagogies and realities. In: Blutesau, P. \& Jackson, A, (Eds.), Interprofessional Education. Making It Happen (pp. 37-58). Basingstoke: Palgrave Macmillan.

Lemieux-Charles, L., \& McGuire, W. L. (2006). What do we know about health care team effectiveness? A review of the literature. Medical Care Research and Review, 63(3), 263-300.

Luke, R., Solomon, P., Baptiste, S., Hall, P., Orchard, C., Rukholm, E., \& Carter, L. (.2009). Online interprofessional health sciences education: From theory to practice. Journal of Continuing Education in the Health Professions, 29(3), 161-167.

Media Education Development Centre. (2006). A survey about education with IT such as e-learning. Tokyo: Media Education Development Centre, 29-30.

Mima, N., \& Yamauchi, Y. (2005). Designing "future learning." Space, activities and community. Tokyo: University of Tokyo Press.

Ministry of Education, Culture, Sports, Science and Technology (2001). Notification: Enforcement of ministerial ordinance to revise part of university establishment standard. Retrieved from http://www.mext.go.jp/b_menu/hakusho/nc/t 19910624001/t19910 $624001 . \mathrm{html}$

Ministry of Education, Culture, Sports, Science and Technology. (2013). The creation of "Specialized occupation practice" pioneering trials which use the framework specialized occupation practice $\sim$ July 12th, 2013. Research Cooperation Conference for Assurance and Improvement of Quality of Vocational Schools. Retrieved from http://www.mext.go.jp/component/b_menu/shingi/ toushin/__icsFiles/afieldfile/2011/01/31/1301878_5_1.pdf.

Ministry of Health, Labour and Welfare (2016). Integrated Community Care System: To implementation Integrated Community Care System. Retrieved from http://www.mhlw.go.jp/stf/seisakunitsuite/bunya/hukushi_kaigo/k aigo_koureisha/chiiki-houkatsu/. 
Ogawa, S., Takahashi, Y., \& Miyazaki, M. (2015). The Current Status and Problems with the Implementation of Interprofessional Education in Japan: An Exploratory Study. Journal of Research in Interprofessional Practice and Education, 5(1), 1-15. Retrieved from http://www.jripe.org/index.php/journal/article/viewArticle/ 181 .

Oizumi. K. (2007). Aging Asia. The time when the conditions of prosperity change. Tokyo: Chuou kouron shinsha.

Oshima, N. (2009). A survey on the recognition and the potential for development of interprofessional education (IPE) at universities in the areas of health care and social service in Japan. Hoken Iryou Fukushi Rennkei, 1(1), 27-33.

Rafter, M. E., Pesun, I. J., Herren, M., Linfante, J. C., Mina, M., Wu, C. D., \& Casada, J. P. (2006). A preliminary survey of interprofessional education. Journal of Dental Education, 70(4), 417-427.

Subcommittee on Elderly Health, Committee on Health/Human Life Science, Science Council of Japan. (2011). Towards interprofessional education for supporting the community$d$ welling elderly. Retrieved from http://www.scj.go.jp/ja/info/kohy o/pdf/kohyo-21-t133-2.pdf

T sukahara, S. (2005). New development and role of the technical school. Nippon roudoukennkyuuzasshi (in Japanese), 542, 70-80. Retrieved from http://web.jil.go.jp/institute/zassi/backnumber/20 05/09/pdf/070-080.pdf.

The Interprofessional Curriculum Renewal Consortium, Australia (2013). Interprofessional health education: A national audit. Retrieved from http://umanitoba.ca/programs/interprofessional /media/IPE_National_Audit_Report_Australia_2013(1).pdf.
Thistlethwaite, J., \& Moran, M. (2010). Learning outcomes for int erprofessional education (IPE): Literature review and synthesis. Journal of Interprofessional Care, 4(5), 503-513.

Thistlethwaite, J., Kumar, K., Moran, M., Saunders, R., Carr, S. (2015). An exploratory review of pre-qualification interprofessional education evaluations. Journal of Interprofessional Care, 29(4), 292-297.

World Health Organization [WHO] (1988). Learning together to work together for health. Report of a WHO study group on multiprofessional education of health personnel: The team approach. (World Health Organisation Techical Report Series 769:1-72). Retrieved from http://apps.who.int/iris/handle/10665/ 37411 .

Yodosha Meibo Henshushitsu (Yodosha Directory Editing Group) (Ed.). (2013). Iiku Kikan Meibo 2013-'14. (Directory of Medical Institutions 2013-'14). Tokyo: Yodosha.

*Michiko Goto, M.A., Ph.D

Assistant Professor

Department of Education and Research in

Family and Community Medicine

Mie University Graduate School of Medicine

2-174 Edobashi, T su 514-8507 Mie JAPAN

Phone: +81-59-231-9087

Fax: +81-59-231-5289

Email:tamgoto@clin.medic.mie-u.ac.jp 